\title{
The Effect of Environmental Treatments on Fiber Surface Properties and Tensile Strength of Sugar Palm Fiber Reinforced Epoxy Composites
}

\begin{abstract}
Fiber glass has been used widely in manufacturing industries, especially marine industries, because of low cost and high strength. However, glass fiber can cause acute irritation to the skin, eyes, and upper respiratory tract. This study looked at the possibility of substituting glass fiber with natural fiber in composite materials. The surface properties of sugar palm fiber (Arenga pinnata) were modified using seawater and freshwater as treatment substances. This led to biological, chemical, and water degradation of the sugar palm fiber. Morphological and structural changes in the fibers were investigated using a scanning electron microscope (SEM). A series of tensile tests based on ASTM D638-99 was carried out on epoxy composites with $15 \%$ sugar palm fiber by volume. It was found that seawater and freshwater treatments improved the surface properties of the sugar palm fiber and thus resulted in better adhesion quality as compared to untreated fiber. An improvement in tensile strength also supported this finding. Treatment with seawater for 30 days proved to be the best, with $67.26 \%$ increase in tensile strength.
\end{abstract}

Keyword: Arenga pinnata fiber, Epoxy composite, Sugar palm fiber, Surface treatment 